\title{
RESEARCH ON THE DYNAMICS OF FLEXIBLE MANIPULATORS
}

\author{
Li Tu', Jinlian Deng ${ }^{1,}$, Huanya Cao ${ }^{1,}$,Pixuan Zhou $^{2}$ \\ ${ }^{\prime}$ Department of Mechanical Engieering, Zhejiang Mechanical\&Electrical College, China; \\ Email: tu li22@vahoo.com.cn. ${ }^{2}$ School of System and Industrial Engieering. University of \\ Arizona, USA.
}

\begin{abstract}
The dynamics of a Flexible Manipulator system are described by an infinitedimensional mathematical model, since the model consists of partial differential equations. But to design a finite-dimensional controller, a finitedimensional system model is needed. To achieve this goal, a finite dimensional approximation needs to be used to model a flexible manipulator, that is, to retain a finite number of modes, and to drop off the other, less significant modes based on the requirements of the controller. The scheme in developing a mathematical model is to use the Lagrangian method or Hamiltonian's Principle to the total kinetic energy, total potential energy and virtual work done by the torque actuated to the joint. This method will not introduce extra errors into system and will be used to obtain the state-space model for a flexible manipulator suggested in this paper. Dynamics of flexible manipulators with shear force and rotatory inertia are derived, and state-space equations with the integration of DC motor dynamics are developed as a theoretical base for mechatronic designs.
\end{abstract}

Key words: Dynamics, Flexible Manipulator; Mathematical Model.

\section{INTRODUCTION}

In last decades, researches on flexible manipulators have increased dramatically. From the perspective of control, recent researches were found in [1] [2] [3] [4] and references therein. These studies did control-intensive work to improve beam performance. But they ignored the impact applied from the beam mechanic shape. The resulting system was only local optimal. In the other hand, a better beam shape to move faster but for less vibration is another topic. One of the first papers on optimal shape for flexible

This project is supported by the National Natural Science Foundation of China under the grant No. 50275091 .

Please use the following format when citing this chapter:

$\mathrm{Tu}, \mathrm{Li}$, Deng, Jinlian, Cao, Huanya, Zhou, Pixuan, 2006, in International Federation for Information Processing (IFIP), Volume 207, Knowledge Enterprise: Intelligent Strategies In Product Design, Manufacturing, and Management, eds. K. Wang, Kovacs G., Wozny M., Fang M., (Boston: Springer), pp. 217-223. 
manipulators is [5] by Karihaloo and Niordson. Extensive studies in optimal shape design have been conducted recently by Wang's group.Wang (1991) [6] simplified substantially the scheme presented in [5]. A minimum weight design of flexible manipulators was developed by Wang and Russell (1992, 1993) [7] [8]. In 1995, they also investigated a new approach [9], the segmentized scheme of optimal design, which treats the flexible beam as a collection of small lengths of rigid beam constrained by each other's interfacial conditions. The new technique converts the optimal shape design problem into a matrix determinant problem. These studies focused on openloop design. They only concerned the beam mechanic construction that is proper design the flexible beam shape can make it suffering less vibration. But in reality, all manipulators must be in closed-loop to get high performance. The couplings between the controller and construction were not considered at larger in these designs.

\section{DYNAMICS OF FLEXIBLE MANIPULATORS}

The dynamics of a Flexible Manipulator system are described by an infinite-dimensional mathematical model, since the model consists of partial differential equations. But to design a finite-dimensional controller, a finitedimensional system model is needed. To achieve this goal, a finite dimensional approximation needs to be used to model a flexible manipulator, that is, to retain a finite number of modes, and to drop off the other, less significant modes based on the requirements of the controller. The $N$-mode expansion for the beam displacement $w(x, t)$ can be given by

$$
w(x, t)=\sum_{i=1}^{N} \psi_{i}(x) q_{i}(\mathrm{t})
$$

The separability in this case refers to describing the displacement as a series of terms which are products of two separate functions, each of that is a function of a single variable, a spatial variable $\mathrm{x}$, and time $\mathrm{t}$, respectively. $i$ is the $i$-th modal shape [10], or eigenfunction. $q_{i}$ is the corresponded generalized modal coordinate describing the flexible deformation.

The scheme in developing a mathematical model is to use the Lagrangian method or Hamiltonian's Principle to the total kinetic energy, total potential energy and virtual work done by the torque actuated to the joint. This method will not introduce extra errors into system and will be used to obtain the state-space model for a flexible manipulator suggested in this paper. 


\subsection{State-space equations of flexible manipulators}

The hub kinetics is written as,

$$
T_{h}=\frac{1}{2} I_{h}\left[\dot{\theta}^{2}+\dot{\omega}^{2}(0, t)^{\prime}\right]
$$

where $\omega=\partial \omega / \partial x=\omega^{\prime}$. After applying Eq. 1 into Eq. 2,

$$
T_{h}=\frac{1}{2} I_{h} \dot{\theta}^{2}+\frac{1}{2} I_{h} \sum_{i=1}^{N} \sum_{j=1}^{N} \dot{q}_{i} \dot{q}_{j} \psi_{i}^{\prime}(0) \psi_{j}^{\prime}(0)
$$

In the same way, the kinetics of the tip load is,

$$
T_{p}=\frac{1}{2} \Gamma_{1} \dot{\theta}^{2}+\dot{\theta} \sum_{\mathrm{i}=1}^{\mathrm{N}} \dot{q} \Gamma_{2}(i)+\frac{1}{2} \sum_{i=1}^{N} \sum_{j=1}^{N} \dot{q}_{i} \dot{q}_{j} \Gamma_{3}(i, j)
$$

where,

$$
\begin{aligned}
& \Gamma_{1}=\left(M_{p} L^{2}+J_{p}+2 M_{p} L a_{c}, \Gamma_{2}(i)=\left(J_{p}+M_{p} L a_{c}\right) \psi_{i}^{\prime}(L)+\left(L+a_{c}\right) M_{p} \psi \psi_{i}(L),\right. \\
& \Gamma_{3}(i, J)=J_{P} \psi \psi_{i}^{\prime}(L) \psi_{j}^{\prime}(L)+M_{p} \psi_{i}(L) \psi_{j}(L)+2 a_{c} \psi \psi_{i}^{\prime}(L) \psi_{j}(L)
\end{aligned}
$$

The kinetic energy of the link,

$$
T_{L}=\frac{1}{2} \Lambda_{1} \dot{\theta}^{2}+\dot{\theta} \sum_{\mathrm{i}=1}^{\mathrm{N}} \dot{q} \Lambda_{2}(i)+\frac{1}{2} \sum_{i=1}^{N} \sum_{j=1}^{N} \dot{q}_{i} \dot{q}_{j} \Lambda_{3}(i, j)
$$

where

$$
\Lambda_{1}=\int_{j}^{L}\left(x^{2}+S\right) \rho d x, \Lambda_{2}(i)=\int_{j}^{L}\left[x \psi_{i}(x)+S \psi_{i}^{\prime}(x)\right] \rho d x, \Lambda_{3}(i, j)=\int_{j}^{L}\left[\psi_{i}(x) \psi_{j}(x)+S \psi_{i}^{\prime}(x) \psi_{j}^{\prime}(x)\right] \rho d x
$$

$S$ is the function of cross section area of the link. The total potential energy is

$$
P=\frac{1}{2} \int_{0}^{L} E I(x) \omega^{2}(x)^{\prime \prime} d x=\frac{1}{2} \sum_{i=1}^{N} \sum_{j=1}^{N} q_{i} q_{j} k(i, j)
$$

where $k(i, j)=\int_{0}^{L} E I(x) \psi_{i}^{\prime \prime}(x) \psi_{j}^{\prime \prime}(x) d x$

The generalized virtual work is derived as, 


$$
W=\tau v^{\prime}(0, t)=\tau \theta+\tau \omega^{\prime}(0, t)=\tau \theta+\tau \sum_{i=1}^{N} \psi_{i}^{\prime}(0) q_{i}(t)
$$

In order to apply the Hamiltonian Principle, let us substitute Eqs. 3, 4, 6, 7,8 for the following formula and group it in terms of $\theta, \delta q_{i}$, and their derivatives,

$$
\Xi=\frac{1}{2} \Omega_{1} \dot{\theta}^{2}+\dot{\theta} \sum_{i=1}^{N} \dot{q}_{i} \Omega_{2}(i)+\frac{1}{2} \sum_{i=1}^{N} \sum_{j=1}^{N} \dot{q}_{i} \dot{q}_{j} \Omega_{3}(i, j)+\tau \theta+\tau \sum_{i=1}^{N} \psi_{i}^{\prime}(0) q_{i}-\frac{1}{2} \sum_{i=1}^{N} \sum_{j=1}^{N} q_{i} q_{j} k(i, j)(10)
$$

where,

$$
\Omega_{1}=I_{h}+\Gamma_{1}+\Lambda_{1}, \Omega_{2}(i)=\Gamma_{2}(i)+\Lambda_{2}(i), \Omega_{3}(i, j)=I_{h} \psi_{i}^{\prime}(0) \psi_{j}^{\prime}(0)+\Gamma_{3}(i, j)+\Lambda_{3}(i, j)
$$

After applying the Hamiltonian method, we obtain, $\delta \Xi=\Omega_{1} \dot{\theta} \dot{\theta} \dot{\theta}+\sum_{i=1}^{N} \Omega_{2}(i) \dot{q}_{i} \dot{\delta} \dot{\theta}+\dot{\theta} \sum_{i=1}^{N} \Omega_{2}(i) \dot{q_{i}}+\sum_{i=1}^{N} \sum_{j=1}^{N} \dot{q}_{1} \Omega_{3}(i, j) \delta \dot{q}_{1}+\tau \delta \theta+\tau \sum_{i=1}^{N} \psi_{i}^{\prime}(0) \delta_{q_{t}}-\sum_{i=1}^{N} \sum_{j=1}^{N} q_{j} k(i, j) \delta q_{i}(12)$



The Hamilton Extended Principle results in, $\int_{t_{0}}^{t_{f}} \delta E d t=\int_{t_{0}}^{t_{f}}\left\{\left[-\Omega_{1} \ddot{\theta}-\sum_{i=1}^{N} \ddot{q}_{i} \Omega_{2}(i)+\tau\right] \delta \theta-\left[\ddot{\theta} \sum_{i=1}^{N} \Omega_{2}(i)+\sum_{i=1}^{N} \sum_{j=1}^{N} \ddot{q}_{j} \Omega_{3}(i, j)+\sum_{i=1}^{N} \sum_{j=1}^{N} q_{j} k(i, j)-\tau \sum_{i=1}^{N} \psi_{i}^{\prime}(0)\right] \varepsilon_{I_{i}}\right\} d t=0,(14)$

These coefficients of $\delta \theta, \delta q_{i}, 1 \leq i \leq N$ must be zero, that is,

$$
\begin{aligned}
& \Omega_{1} \ddot{\theta}+\sum_{i=1}^{N} \ddot{q}_{i} \Omega_{2}(i)-\tau=0 \\
& -\ddot{\theta} \Omega_{2}(i)-\sum_{j=1}^{N} \ddot{q}_{j} \Omega_{3}(i, j)-\sum_{j=1}^{N} q_{j} k(i, j)+\tau \psi_{i}^{\prime}(0)=0, \quad 0 \leq i \leq N,
\end{aligned}
$$

or in matrix form, they are,

$$
M \ddot{x}+k x=b \tau
$$

where, 


$$
\begin{aligned}
& x=\left[\theta, q_{1}, q_{2}, q_{3}, \ldots, q_{N}\right]_{(N+1,1)}^{T}, \quad b=\left[1, \psi_{1}^{\prime}(0), \psi_{2}^{\prime}(0), \psi_{3}^{\prime}(0), \ldots, \psi_{N}^{\prime}(0),\right]_{(N+1, \mathrm{I})}^{T^{T}} \text {, } \\
& M=\left[\begin{array}{ccccc}
\Omega_{1} & \Omega_{2}(1) & \Omega_{2}(2) & \cdot & \Omega_{2}(N) \\
\Omega_{2}(1) & \Omega_{3}(1,1) & \Omega_{3}(1,2) & \cdot & \Omega_{3}(1, N) \\
\cdot & \cdot & \cdot & \cdot & \cdot \\
\Omega_{2}(N) & \Omega_{3}(N, 1) & \Omega_{3}(N, 2) & \cdot & \Omega_{3}(N, N)
\end{array}\right] \\
& K=\left[\begin{array}{ccccc}
0 & 0 & 0 & \cdot & 0 \\
0 & k(1,1) & k(1,2) & \cdot & k(1, N) \\
\cdot & \cdot & \cdot & \cdot & \cdot \\
0 & k(N, 1) & k(N, 2) & . & k(N, N)
\end{array}\right]
\end{aligned}
$$

As has been shown, $\Omega_{3}(i, j)=\Omega_{3}(j, i), k(i, j)=k(j, i)$, thus the matrices $M, K$ are symmetric and named mass and rigidity matrices respectively.

Next, the actuator dynamics need to be incorporated into the link system. It is assumed

that the arm is driven by a permanent magnet DC-motor. Therefore, the actuator dynamics can be described as,

$$
-J_{m} \ddot{\theta}-\left(B_{m}+\frac{K_{b} K}{R}\right) \dot{\theta}+\frac{K_{m}}{R} v_{c}=\tau
$$

where $J_{m}$ is the actuator inertia, $B_{m}$ the friction coefficient, $K_{m}$ the torque constant, $K_{b}$ the back emf constant, $R$ the armature resistance, and $\theta, v_{c}$ the hub rotation and armature voltage, respectively. In general, all motor circuit parameters can be considered as design variables. The overall state variable is defined as,

$$
q=\left[\begin{array}{l}
x \\
\dot{x}
\end{array}\right]
$$

Combining Eq. 17 with Eq. 18, we present the overall system state space equations as,

$$
q=A \dot{q}+B, \quad u=v_{c},
$$

where

$$
\begin{aligned}
& A=\left[\begin{array}{cc}
0 & I \\
-\bar{M}^{-1} K & -\bar{M}^{-1} \bar{B}
\end{array}\right], \quad B=\left[\begin{array}{c}
0 \\
\bar{M}^{-1} \bar{D}
\end{array}\right], \quad \text { (22) } \\
& \bar{M}=\left(M+J_{m} \underline{e}_{1}\right)^{-1}, \bar{B}=\left(B_{m}+\frac{K_{b} K_{m}}{R}\right) b \underline{e_{1}}, \quad \bar{D}=b \frac{K_{m}}{R}, \underline{e_{1}}=\left[\begin{array}{llll}
1 & 0 & \ldots & 0
\end{array}\right]_{1 \times n}
\end{aligned}
$$


$A$ is the function of beam construction, thus any changes in beam mechanical shape will result in a different $\mathrm{A}$, which provides the basis for simultaneously optimal construction and control based on the mechatronic formulation discussed early.

\subsection{Output specifications}

As can be seen from the system state-space equations, the state vector consists of $\theta$, the generalized modal coordinates $q_{i}$, and their first order derivatives. So all controllers based on the state feedback are indirect, which means that the states need to be predicted. This will trade off with computation time and bring one more inaccuracy into a closed-loop, cutoff closed-loop bandwidth. This presents hindrances to real-time processing and high motion speed. Output feedback takes precedence over state space feedback as far as the mechatronic approach and objectives of this paper are concerned.

Tip deflection output, which is,

$$
y=w(L, t)=\sum_{i=1}^{N} \psi_{i}(L) q_{i}=\left[\begin{array}{llllll}
0 & \psi_{1}(L) & \psi_{2}(L) & \ldots & \psi_{N}(L) & 0
\end{array}\right] q,
$$

where $\psi_{i}$ is the ith eigenfunction, $\overline{0}_{1 \times(n+1)}$ is zero vector. A CCD camera clipped on the hub can be used to measure the output.

The tip position output is,

$$
y=v(L, t)=L \theta+\sum_{i=1}^{N} \psi_{i}(L) q_{i}=\left[\begin{array}{llllll}
L & \psi_{1}(L) & \psi_{2}(L) & \ldots & \psi_{N}(L) & 0
\end{array}\right] q
$$

The hub tangent angle output is,

$$
y=\Theta=\theta+\sum_{i=1}^{N} \psi_{i}^{\prime}(0) q_{i}=\left[\begin{array}{llllll}
1 & \psi_{1}^{\prime}(0) & \psi_{2}^{\prime}(0) & \ldots & \psi_{N}^{\prime}(0) & 0
\end{array}\right] q
$$

A potentiometer may be used for measuring $\Theta$.

In this mechatronic design, output feedback maybe one of above outputs, or the mixture,

such as,

$$
\begin{aligned}
& y=C q=\left[\begin{array}{c}
w(L, t) \\
v^{\prime}(L, t)
\end{array}\right] q, \\
& C=\left[\begin{array}{lllll}
0 & \psi_{1}(L) & \psi_{2}(L) & \psi_{N}(L) & 0 \\
1 & \psi_{1}^{\prime}(0) & \psi_{2}^{\prime}(0) & \psi_{N}^{\prime}(0) & 0
\end{array}\right]
\end{aligned}
$$


The corresponding state-variable equations are,

$$
\dot{q}=A q+b u, \quad \mathrm{y}=C q .
$$

\section{CONCLUSION}

Mechatronic design is a global optimization of the overall system. For a flexible manipulator, the overall system is the integration of link dynamics, DC motor equation, measuring sensors, and the selected controller. As above, these output feedbacks can be used in control design to make the improvement of the suggested mechatronic approach and to make comparison between them .

\section{REFERENCES}

1. J. Doyle, K. Glover(1989), "State-Space Solution to Standard H2 and H Control Problem", IEEE AC, Vol. 34, No. 8 , pp. 831-847.

2. B. Francis, A Course in H Control Theory, (Springer-Verlag, Berlin-New York, 1998).

3. L. B. Guti errez, F. L. Lewis, J. A. Lowe (1998), Implementation of a Neural Network tracking Controller for a Single Flexible link: Comparison with PD and PID Controller, in IEEE Trans. on Industrial Electronics, Vol. 45, No. 2.

4. A. Jnifene, A. Fahim (1997), A Computed Torque/Time Delay Approach to the End-Point Control of a One-Link Flexible Manipulator, Dynamics and Control, Vol. 7, pp. 177-189.

5. M. Fujita, et al (1991), "Experimental Evaluation of H Control for a Flexible Beam Magnetic Suspension System," S. Hosoe (ed) Robust Control, Pro of a workshop held in Toyko, June 23-24.

6. F. L. Lewis, V. L. Syrmos (1995), Optimal Control, John Wiley and Sons, Inc., Second Edition.

7. K. Lezn, H. Ozbay, et al (1991), Frequency Domain Analysis and Robust Control Design for an Ideal Flexible Beam, Automatica, Vol. 27, pp. 947-961.

8. A. Tchernychev, A. Sideris, J. Yu (1997), Constrained $H$ Control of an Experimental Flexible Link, Trans. o fthe ASME, June.

9. Fei-Yue Wang (1994), On the Extremal Fundamental Frequencies of One-Link Flexible Manipulators, International Journal of Robotics Research, Vol. 13, No. 2, pp. 162-170.

10.Fei-Yue Wang and J.L.Russell(1992), Optimum Shape Constructure of Flexible Manipulators with Tip Loads, Proceedings of IEEE Intl,Conf. on Decision and Contral(Tucson,Arizona), Vol. 1, pp. 311-316. 